\title{
El cáncer ahora
}

\section{Cancer now}

\author{
Raquel Gerson Cwilich*
}

Citar como: Gerson CR. El cáncer ahora. An Med ABC. 2021; 66 (4): 236-237. https://dx.doi.org/10.35366/102922

Planear es traer el futuro al presente para poder hacer algo por el ahora. Alan Lakein

En los próximos años se visualiza un incremento en la incidencia del cáncer debido a los cambios epidemiológicos asociados al envejecimiento de la población y un aumento en la prevalencia como resultado de diagnósticos tempranos y de tratamientos eficaces, esto a nivel global impondrá retos importantes en la demanda de atención de los servicios sanitarios de pacientes con cáncer. ${ }^{1}$

Las nuevas terapias han propiciado una importante reducción en la tasa de mortalidad mundial. Dos de tres personas con cáncer sobrevivirán más de cinco años ${ }^{2}$ y seis de 10 enfermos de cáncer se curan, cifras que van en aumento. ${ }^{3}$ Se calcula que entre $30 \mathrm{y}$ $50 \%$ de los tipos de cáncer son prevenibles mediante la adopción de hábitos saludables, vacunación, diagnóstico y tratamientos oportunos. ${ }^{4}$

Se prevé un aumento de $35 \%$ de nuevos casos para $2030 .^{4}$

La investigación del cáncer muestra ser la mejor oportunidad de superar la práctica de la medicina del hoy y la del futuro. La investigación salva vidas, es la mejor inversión para los pacientes con cáncer.

Actualmente el experto en manejar pacientes con diagnósticos oncológicos tiene un enorme reto ante la mayor demanda de atención de la sociedad frente a la rápida evolución de los nuevos conceptos sobre la biología tumoral, y de los avances en investigación que generan mayor conocimiento mediante estrategias de colaboración, diseño, medicina traslacional e innovación. La innovación no se detiene, existen más de 7,000 medicamentos en diversas fases de desarrollo, ${ }^{4}$ con la mentalidad dirigida hacia personalizar los tratamientos. La medicina de precisión es ya una promesa y un gran reto en la lucha contra el cáncer.

La clasificación tradicional de las enfermedades neoplásicas se hace en función del aparente órgano de origen, se procede a considerar los datos morfológicos para arribar ulteriormente a las características citogenéticas. Hoy en día, para maximizar las probabilidades de ofrecer un tratamiento eficaz el cáncer debe ser catalogado y clasificado con base en sus propiedades moleculares. La medicina personalizada requiere estrecha colaboración de un equipo multidisciplinario de especialistas ${ }^{5}$ para poder comprender mejor cada caso. Estamos cerca, pero aún falta mucho por entender y más por hacer para lograr que este nuevo conocimiento llegue a todos los pacientes.

La inmunoterapia es una modalidad innovadora en el tratamiento del cáncer que busca reactivar un sistema de inmunovigilancia que ha fallado ante los diversos mecanismos de escape que las células tumorales han establecido para lograr proliferar. Los nuevos anticuerpos monoclonales dirigidos contra los puntos de control inmunológico han ingresado a la práctica clínica en diversos tumores, pero aún falta

\footnotetext{
* Directora del Centro de Cáncer, Departamento de Oncología Médica. Centro Médico ABC, IAP Instituciones de Asistencia Privada, Ciudad de México.
} 
mayor conocimiento para poder incrementar el potencial terapéutico que esta clase de medicamentos pueda lograr; ${ }^{6}$ en la actualidad se estudian nuevos experimentos.

Desde una visión epidemiológica, la prevención del cáncer puede tener mayor impacto en la perspectiva global, para ello se debe buscar una amplia y continua educación de la población.

La identificación de las poblaciones de mayor riesgo de padecer una enfermedad oncológica va en aumento con la aplicación de las medidas más apropiadas de diagnóstico y referencia oportuna. ${ }^{7}$ Sin embargo, la sociedad debe tener la suficiente conciencia para cambiar hábitos asociados al cáncer, esta trascendente medida bajaría la incidencia de varios tumores.

La oncología global como comunidad científica ha experimentado un crecimiento muy importante acompañado de un aumento en el interés, capacidad y oportunidad de cimentar fundamentos sólidos para los descubrimientos del futuro. Permite redoblar esfuerzos en la diseminación del conocimiento y dedicación para erradicar el cáncer como se conoce hasta el día de hoy. ${ }^{8}$

\section{POSICIÓN DEL CENTRO DE CÁNCER ABC EN EL TRATAMIENTO}

El cáncer es una enfermedad muy horizontal que requiere de una institución de sólidos cimientos y valores con lo último en tecnología y líderes expertos en el manejo de diferentes tumores, que sustente la formación de recursos humanos mediante la disciplina académica, una institución que cree programas de investigación clínica y traslacional «estado del arte», un centro de atención especializada con la cultura que beneficie a los pacientes. ${ }^{9}$

El Centro de Cáncer ABC se seguirá enfocando en proveer educación al público, información en pro- gramas de prevención y detección para lograr mayor impacto a largo plazo y crear conciencia de atención oportuna.

Con la visión de desarrollar los avances de la ciencia, una atención médica de vanguardia y lograr la meta de una calificación excepcional en el día a día por parte de nuestros pacientes.
"Futuro no es lo que viene, sino lo que se va hacer». Raquel Gerson Cwilich

\section{REFERENCIAS}

1. Pilleron S, Soto-Perez-de-Celis E, Vignat J, Ferlay J, Soerjomataram I, Bray F et al. Estimated global cancer incidence in the oldest adults in 2018 and projections to 2050. Int J Cancer. 2021; 148 (3): 601-608.

2. CDC. Comunicado de Prensa; [Citado el: Jueves, 12 de marzo de 2015]; https://www.cdc.gov/spanish/mediosdecomunicacion/ comunicados/p_supervivencia_cancer_031215.html

3. Europapress. Casi seis de cada 10 enfermos de cáncer se curan, según experto; [Publicado: 03/02/2020] ; Disponible en: https://www.europapress.es/cantabria/noticia-casi-seis-cada10-enfermos-cancer-curan-experto-20200203131902.html

4. AMIIF. Cuatro ejes que guían la investigación y el desarrollo de terapias innovadoras; [Citado el: diciembre 9, 2019]; Disponible en: https://amiif.org/cuatro-ejes-que-guian-lainvestigacion-y-el-desarrollo-de-terapias-innovadoras

5. Agur Z, Elishmereni M, Kheifetz Y. Personalizing oncology treatments by predicting drug efficacy, side-effects, and improved therapy: mathematics, statistics, and their integration. Wiley Interdisciplinary Reviews: Syst Biol Med. 2014; 6 (3): 239-253.

6. Helmy KY, Patel SA, Nahas GR, Rameshwar P. Cancer immunotherapy: accomplishments to date and future promise. Ther Deliv. 2013; 4 (10): 1307-1320.

7. Vineis P, Wild CP. Global cancer patterns: causes and prevention. Lancet. 2014; 383 (9916): 549-557.

8. Gopal S, Sharpless NE. Cancer as a global health priority. JAMA. 2021.

9. Kaasa S, Loge JH, Aapro M, Albreht T, Anderson R, Bruera $\mathrm{E}$ et al. Integration of oncology and palliative care: a Lancet Oncology Commission. Lancet Oncol. 2018; 19 (11): e588-e653. 